\title{
Study of an Industrial Electrode Dryer of a Lithium-Ion Battery Manufacturing Plant: Dynamic Modeling
}

\author{
Emil Oppegård ${ }^{1} \quad$ Asanthi Jinasena $^{2} \quad$ Anders Hammer Strømman $^{2} \quad$ Jon Are Suul $^{1,3}$ Odne Stokke \\ Burheim $^{2}$ \\ ${ }^{1}$ Department of Engineering Cybernetics, Norwegian University of Science and Technology, Norway, \\ emilop@stud.ntnu.no, jon.are.suul@ntnu.no \\ ${ }^{2}$ Department of Energy and Process Engineering, Norwegian University of Science and Technology, Norway, \\ \{asanthi.jinasena, anders.hammer.stromman, odne.s.burheim\} @ntnu.no \\ ${ }^{3}$ SINTEF Energy Research, Norway
}

\begin{abstract}
A dynamic model for lithium-ion battery (LIB) electrode manufacturing and drying is developed in this paper. The model is intended for analysis of different drying technologies, energy requirement calculations, and optimization and control of the drying process. The model shows that the infrared drying is faster than the convective drying when the heat source temperature is the same. The energy required to evaporate the solvent can be reduced by gradually changing the hot air temperature. Drying is the most energy-intensive process in cell manufacturing, and the cell manufacturing process is the biggest contributor to greenhouse gas emissions in the LIB industry. Therefore, the presented model is useful for accurate estimation of the environmental impact as well as for identifying the appropriate measures to reduce energy requirements in the rapidly growing LIB industry.

Keywords: lithium-ion battery, electric vehicle, electrode drying, convection, infrared radiation, sustainable energy, model, control
\end{abstract}

\section{Introduction}

Efforts to decarbonize the transport sector are currently leading to a shift from fossil fuelled vehicles to electric mobility. Although this development is still at an early stage, the global market for electric mobility is expected to grow rapidly in the future. Currently, the largest uptakes of electric vehicles (EVs) are appearing in China and Europe. Within Europe, the Nordic countries have a leading position. Norway has a high share of EVs, where $40 \%$ of all new car sales in 2019 were battery electric vehicles (BEVs) (International Energy Agency, 2019).

The demand for LIB is expected to increase rapidly with the emerging technology for electric mobility. Ensuring that this development fulfills its potential for climate change mitigation is important. In that regard, life cycle assessment studies provide good insights. They have shown that the carbon footprint from LIB production may contribute to a significant part of the overall greenhouse gas emissions of BEVs (Kurland, 2020; Ellingsen et al.,
2016). Especially, energy requirements in the cell manufacturing process are important drivers for these emissions (Kurland, 2020).

A thorough understanding of the energy requirements of the cell manufacturing process is crucial for an accurate assessment of the current practices, the identification of appropriate measures to reduce energy requirements, and the maximization of the potential for mitigating climate change. Thus, there is a need for research driving towards sustainable, low cost, efficient, and high energy density battery manufacturing practices. Consequently, the use of sustainable materials, the adaption of less energy-intensive technologies, and energy benchmarking of process control practices are becoming important (Susarla et al., 2018). An in-depth understanding of the underlying principles of battery manufacturing would give valuable insight into the fulfillment of these requirements.

The LIB consists of electrodes (cathode and anode), separator, electrolyte, and a casing, for more details see Burheim (2017). Both the cathode and anode are made of active materials, carbon black additives, and a polymeric binder. Usually, the active material for the cathode is lithium metal oxides such as $\mathrm{LiMn}_{2} \mathrm{O}_{4}, \mathrm{Li}_{\mathrm{a}} \mathrm{Ni}_{\mathrm{x}} \mathrm{Mn}_{\mathrm{y}} \mathrm{Co}_{\mathrm{z}} \mathrm{O}_{2}$. For either cathode or anode, carbon black conductive additives are acetylene black, or graphite while the polymeric binder is polyvinylidene difluoride (Kwade et al., 2018). These materials are dry-mixed together and combined with a solvent to make a slurry. The solvent is either N-Methyl-2pyrrolidone (NMP), or water. Once the slurry is made, it is coated onto a thin aluminium (cathode slurry) or copper (anode slurry) foil, typically of $25-35 \mu \mathrm{m}$ in thickness (Burheim, 2017). These coated thin films are then dried to remove most of the solvent. The dried films are then pressed to gain the predefined porosity and thickness, which is known as calendaring. Then inside a dry room, the electrodes are cut, stacked, and assembled into the cell, with the rest of the parts. This cell is then activated by cell conditioning processes.

Out of these, the most energy-intensive step is the elec- 
trode drying process (Yuan et al., 2017; Dai et al., 2019), which also highly influences the cell performance by the drying rate and method (Ahmed et al., 2016). A physicsbased model of this process would not only give a good insight into dynamic drying behavior and energy optimization, but also the potential of using new materials, different compositions, and different drying techniques. Although modeling of thin-layer drying is a well-researched topic in food, paper, paint, and pharmaceutical industries, there is limited available literature dedicated to LIB manufacturing industry. The few existing models (Ahmed et al., 2016; Susarla et al., 2018) are insufficient to fulfill the above requirements. Therefore, we develop a dynamic mathematical model of the electrode drying process. The presented model is intended for analysis of different drying technologies, including calculation of energy requirement as well as optimization and control of the process. In addition, a sensitivity analysis of process parameters is presented to evaluate the model performance.

The paper is organized as follows. The system description including the model derivation, the used numerical method and its' stability are presented in Section 2. This is followed by detailed results and discussions being presented in Section 3. Finally the conclusions that are drawn from the study are summarized in Section 4.

\section{Model Development}

Drying processes are inherently complex due to simultaneous mass and heat transfer. The shrinkage of the thin film further complicates the process due to the formation of a porous structure as the solvent evaporates. The drying process has two stages; in the first stage evaporation is only a surface phenomenon and the thin film shrinks. In the second stage, the film does not shrink, evaporation is capillary-driven, hence no longer limited to the surface (Susarla et al., 2018). Further, during the first phase, the active material particles and binder are dispersed in the solvent. However, in the second phase, the particles are drawn close to each other and the voids created by solvent vaporization get replaced by air. Since the evaporation is governed by different phenomena, the two distinct stages require different mathematical descriptions. For this study, the developed model will emphasize only on the first stage.

\subsection{Model Derivation}

To derive a simple, yet accurate model that describes the drying process which later can be used in optimization and control, the problem is simplified by well-established assumptions and simplifications. Prior to the drying process, the slurry is assumed to be uniformly mixed and coated onto the current collectors. Thus, the temperature and slurry distribution in the $x$-direction (width of the film) and in the $y$-direction (length of the film) are uniform within the control volume. The electrode is assumed to be moving in the $y$-direction with a constant velocity. This leaves the control volume to only consider fluxes in the $z$-direction (height of the film), which results in a onedimensional model. For further simplification, the thickness of the film is assumed to be so small that the temperature development in the $z$-direction is uniform.

The fluxes in the $z$-direction due to diffusion can be expressed by the one-dimensional diffusion equation:

$$
\frac{\partial C}{\partial t}=D \frac{\partial^{2} C}{\partial z^{2}}
$$

where, $C$ represents the weight fraction of the solvent, $t$ is the time and $D$ is the diffusion coefficient. The diffusion coefficient is highly dependent on the temperature and concentration. Mesbah et al. (2014) expresses the diffusion coefficient as a function of polymer volume fraction and temperature as follows,

$$
D=D_{0} \cdot\left(\frac{1-\phi_{\mathrm{p}}}{1+\phi_{\mathrm{p}}}\right)^{\gamma} \cdot \exp \left(\frac{-E}{R T}\right) .
$$

where $D_{0}$ refers to the mutual diffusion coefficient and varies with different compositions, $\phi$ is the polymer volume fraction, $E$ is the activation energy, $R$ is the universal gas constant, $\gamma$ is an empirical value and $T$ is the film temperature. The liner is assumed to be impenetrable, which is equivalent to imposing a zero flux boundary condition at the bottom of the film:

$$
z=0: \quad \frac{\partial C}{\partial z}=0
$$

For the boundary at the top of the film, the boundary condition is governed by the evaporation of the solvent. The shrinkage of the film also has to be taken into consideration. Mesbah et al. (2014) expressed the boundary condition as:

$$
z=h(t): \quad D \frac{\partial C}{\partial z}+C \frac{\mathrm{d} h}{\mathrm{~d} t}=-\frac{k_{\mathrm{m}} M_{\mathrm{s}}}{\rho_{\mathrm{s}} R} \cdot\left(\frac{P_{\mathrm{s}}}{T}-\frac{P_{\mathrm{a}}}{T_{\mathrm{a}}}\right)
$$

where, $h$ is the varying thickness of the film, $k_{m}$ is the mass transfer coefficient, $M_{s}$ is the molecular weight of the solvent, $\rho_{s}$ is the density of the solvent, $P_{s}$ is the partial pressure of the solvent, $P_{a}$ is the vapor pressure in the air and $T_{a}$ is the temperature of the heated air. The vapor pressure and temperature of the heated air is considered to be constant throughout the drying process. The vapor pressure is approximated using the Antoine equation:

$$
P_{a}=\varphi \cdot 133.332 \cdot 10^{\left(7.54826-\frac{1979.68}{222.2+\left(T_{a}-273.15\right)}\right)}
$$

where $\varphi$ represents the relative humidity of the heated air. In contrast to the vapor pressure, $P_{S}$ varies with both solvent concentration and temperature. Khansary (2016) expresses the solvent vapor pressure using Flory Huggin's theory for polymeric solutions as follows,

$$
P_{s}=P_{0} \cdot \exp \left(\phi_{\mathrm{p}}^{2} \mathscr{X}+\ln \left(\phi_{\mathrm{s}}\right)+\left(1-\frac{V_{\mathrm{s}}}{V_{\mathrm{p}}}\right) \phi_{\mathrm{p}}\right),
$$


where, $P_{0}$ is the vapor pressure of the pure solvent, $\mathscr{X}$ is the Flory Huggins interaction parameter, $\phi_{\mathrm{s}}$ represents the volume fraction of the solvent, and $V_{\mathrm{s}}, V_{\mathrm{p}}$ are the volumes of solvent and solid, respectively.

An expression for the thickness of the film is obtained by applying mass balance to the system. Since the thickness is solely dependent on the evaporation of the solvent, it can be described by the following formula:

$$
\frac{\mathrm{d} h}{\mathrm{~d} t}=-\frac{k_{\mathrm{m}} M_{\mathrm{s}}}{\rho_{\mathrm{f}} R} \cdot\left(\frac{P_{\mathrm{s}}}{T}-\frac{P_{\mathrm{a}}}{T_{\mathrm{a}}}\right),
$$

where, $\rho_{\mathrm{f}}$ represents the density of the film. The densities of the combined solids and solvent are assumed to be constant throughout the drying process. However, the density of the film is expressed as a linear function of the individual densities and solvent mass fraction:

$$
\rho_{\mathrm{f}}=\rho_{\mathrm{s}} \cdot C+(1-C) \cdot \rho_{\mathrm{p}},
$$

where, $\rho_{\mathrm{p}}$ is the bulk density of the solids.

Energy balance is applied to the system with the stated assumptions. In addition, contributions from surface tension and shear stress gradients are assumed to be so small that they can be neglected without affecting the accuracy significantly. The energy balance for the film is as follows:

$$
h \frac{\mathrm{d} T}{\mathrm{~d} t}+T \frac{\mathrm{d} h}{\mathrm{~d} t}=-\frac{k_{\mathrm{m}} M_{\mathrm{s}} \lambda}{\rho_{\mathrm{f}} C_{\mathrm{p}} R} \cdot\left(\frac{P_{\mathrm{s}}}{T}-\frac{P_{\mathrm{a}}}{T_{\mathrm{a}}}\right)+\frac{\dot{q}_{\text {in }}}{\rho_{\mathrm{f}} C_{\mathrm{p}}}
$$

where, $\lambda$ is the latent heat of vaporization of the solvent and can be approximated by a second degree polynomial as follows:

$$
\begin{aligned}
& \lambda=6.991 \cdot T^{2}-6193 \cdot T+1.848 \cdot 10^{6}: \mathrm{NMP} \\
& \lambda=-3.345 \cdot T^{2}-259.3 \cdot T+2.817 \cdot 10^{6}: \text { water }
\end{aligned}
$$

$C_{\mathrm{p}}$ is the specific heat of the film. The variation of $C_{\mathrm{p}}$ within the temperature range is insignificant. Therefore, it is set to a constant for simplicity. $\dot{q}_{\text {in }}$ represents the heat transferred into the film. The heat transportation is either done by forced convection, radiation or a combination of both depending on the configuration of the drying process. Heat transportation done by convection is given by:

$$
\dot{q}_{\text {in }}=k_{\mathrm{c}} \cdot\left(T_{\mathrm{a}}-T\right)
$$

where, $k_{\mathrm{c}}$ is the heat transfer coefficient. Susarla et al. (2018) determines the heat transfer coefficient based on the air velocity as follows:

$$
k_{\mathrm{c}}=0.037 \cdot v^{0.8} \cdot\left(\frac{\mu}{\rho_{\mathrm{a}}}\right)^{-0.8} \cdot \operatorname{Pr}^{1 / 3} \cdot L^{-0.2}
$$

where, $v$ is the air velocity, $\mu$ is the viscosity of the air, $\rho_{\mathrm{a}}$ is the density of the air, Pr is the Prandtl number and $L$ is the characteristic length. Only the convective heat transfer is considered during convective drying, and the temperature of the hot air is assumed to be unchanged by the effects of the solvent evaporation.

However, for infrared radiative drying, the radiative heat transfer from the source to the film, and the convective heat transfer from the source to the air stream above the film are considered. This is because the airflow rate is considered to be at ambient conditions, hence the temperature of the air changes with heat transfer from the source, and the solvent evaporation. Heat transportation from the source to the film due to radiation can be written as follows,

$$
\dot{q}_{\text {in }}=\sigma \varepsilon \cdot\left(T_{\mathrm{r}}^{4}-T^{4}\right),
$$

where, $T_{\mathrm{r}}$ is the heat source temperature, $\sigma$ is the Stefan Boltzman constant and $\varepsilon$ is the emissivity of the surface of the film. The energy required for solvent evaporation throughout the process is given by:

$$
Q=\int_{0}^{t_{\mathrm{f}}} \dot{m}_{\mathrm{evap}} \cdot \lambda \mathrm{d} t
$$

where, $t_{\mathrm{f}}$ is the final time of the drying process and $\dot{m}_{\text {evap }}$ is the evaporation rate. The total energy requirement for drying would include the energy needed for the heat source, in addition to $Q$.

In addition, the air temperature during the infrared drying can be calculated by the energy balance to a unit volume of air stream as follows,

$$
\frac{\mathrm{d} T_{a}}{\mathrm{~d} t}=\frac{k_{c}\left(T_{\mathrm{r}}+T-2 T_{\mathrm{a}}\right)+\dot{m}_{\mathrm{evap}} C_{\mathrm{p}_{\mathrm{s}}}\left(T-T_{\mathrm{a}}\right)}{\rho_{\mathrm{a}} C_{\mathrm{pa}_{\mathrm{a}}}},
$$

where $C_{\mathrm{p}_{\mathrm{s}}}$ and $C_{\mathrm{p}_{\mathrm{a}}}$ are specific heat capacities of the solvent and air, respectively.

To complete the model, the initial conditions at $t(0)$ are given by:

$$
C(0, z)=C_{0}, \quad T(0)=T_{0}, \quad h(0)=h_{0}
$$

\subsection{Dimensionless Model}

To simplify the numerical approach by immobilizing the otherwise moving boundary conditions at the surface, dimensionless variables are introduced. The variables can be expressed by the following dimensionless variables.

$$
\begin{aligned}
\tilde{t}:=\frac{t}{t_{0}} & \tilde{z}:=\frac{z}{h} & \tilde{h}:=\frac{h}{h_{0}} \\
\tilde{T}:=\frac{T}{T_{0}} & \tilde{C}:=\frac{C}{C_{0}} & \tilde{D}:=\frac{D}{D_{0}}
\end{aligned}
$$

where, $\tilde{z}, \tilde{h}, \tilde{T}, \tilde{C}, \tilde{D}$ represent the dimensionless variables which ranges from 0 to 1 , while $\tilde{t}=t$, by setting $t_{0}$ to unity for simplicity. Substituting equations 18 and 19 into the continuous model yields the following dimensionless model:

$$
\frac{\mathrm{d} \tilde{h}}{\mathrm{~d} \tilde{t}}=-\frac{k_{\mathrm{m}} M_{\mathrm{s}} t_{0}}{\rho_{\mathrm{f}} R h_{0}} \cdot\left(\frac{P_{\mathrm{s}}}{\tilde{T} T_{0}}-\frac{P_{\mathrm{a}}}{T_{\mathrm{a}}}\right),
$$




$$
\begin{aligned}
& \frac{\mathrm{d} \tilde{T}}{\mathrm{~d} \tilde{t}}=-\frac{k_{\mathrm{m}} M_{\mathrm{s}} \lambda t_{0}}{\rho_{\mathrm{f}} C_{\mathrm{p}} R \tilde{h} h_{0}} \cdot\left(\frac{P_{\mathrm{s}}}{\tilde{T} T_{0}^{2}}-\frac{P_{\mathrm{a}}}{T_{\mathrm{a}} T_{0}}\right)-\frac{\tilde{T}}{\tilde{h}} \frac{\mathrm{d} \tilde{h}}{\mathrm{~d} \tilde{t}} \\
&+\frac{t_{0}}{\rho_{\mathrm{f}} C_{\mathrm{p}} \tilde{h} h_{0} T_{0}} \dot{q}_{\mathrm{in}}, \\
& \frac{\partial \tilde{C}}{\partial \tilde{t}}=\frac{\tilde{D} D_{0} t_{0}}{\left(\tilde{h} h_{0}\right)^{2}} \frac{\partial^{2} \tilde{C}}{\partial \tilde{z}^{2}}
\end{aligned}
$$

with the boundary conditions:

$$
\begin{array}{ll}
\tilde{z}=0: & \frac{\partial \tilde{C}}{\partial \tilde{z}}=0 \\
\tilde{z}=1: & \frac{\tilde{D} D_{0} C_{0}}{\tilde{h} h_{0}} \frac{\partial \tilde{C}}{\partial \tilde{z}}+\frac{\tilde{C} C_{0} h_{0}}{t_{0}} \frac{\mathrm{d} \tilde{h}}{\mathrm{~d} \tilde{t}}= \\
& -\frac{k_{\mathrm{m}} M_{\mathrm{s}}}{\rho_{\mathrm{s}} R}\left(\frac{P_{\mathrm{s}}}{\tilde{T} T_{0}}-\frac{P_{\mathrm{a}}}{T_{\mathrm{a}}}\right)
\end{array}
$$

and the initial conditions at $t(0)$ :

$$
\tilde{C}(0, \tilde{z})=1, \quad \tilde{T}(0)=1, \quad \tilde{L}(0)=1 .
$$

\subsection{Numerical Approach and Stability}

The system is solved in MATLAB ${ }^{\circledR}$ using the finite difference method (FDM). FDM approximates the derivatives using the finite part of the Taylor Series expansion (Tannehill et al., 1997). Before applying the FDM, the domain of the system is discretized into a finite grid of nodes, with the nodes representing the system properties at a finite point in both space and time. The forward-time-centralspace (FTCS) differential scheme is used in approximating the derivatives as follows (Tannehill et al., 1997):

$$
\begin{aligned}
\frac{\mathrm{d} f}{\mathrm{~d} \tilde{t}} & =\frac{f^{i+\Delta \tilde{t}}-f^{i}}{\Delta \tilde{t}} \\
\frac{\partial^{2} f}{\partial \tilde{z}^{2}} & =\frac{f_{j+\Delta \tilde{z}-2 f_{j}+f_{j-\Delta \tilde{z}}}}{\Delta \tilde{z}^{2}}
\end{aligned}
$$

Applying the FDM with FTCS-scheme yields the following discrete equations:

$$
\begin{gathered}
\tilde{h}^{i+\Delta \tilde{t}}=-t_{0} \Delta \tilde{t} \frac{k_{\mathrm{m}} M_{\mathrm{s}}}{\rho_{\mathrm{f}} R h_{0}} \cdot\left(\frac{P_{\mathrm{s}}}{\tilde{T}^{i} T_{0}}-\frac{P_{\mathrm{a}}}{T_{\mathrm{a}}}\right)+\tilde{h}^{i} \\
\tilde{T}^{i+\Delta \tilde{t}}=-t_{0} \Delta \tilde{t} \frac{k_{\mathrm{m}} M_{\mathrm{s}} \lambda}{\rho_{\mathrm{f}} C_{p} R \tilde{h}^{i} h_{0}} \cdot\left(\frac{P_{\mathrm{s}}}{\tilde{T}^{i} T_{0}^{2}}-\frac{P_{\mathrm{a}}}{T_{\mathrm{a}} T_{0}}\right)+ \\
\frac{t_{0} \Delta \tilde{t}}{\rho_{\mathrm{f}} C_{\mathrm{p}} \tilde{h}^{i} h_{0} T_{0}} \cdot \dot{q}_{\mathrm{in}}-\frac{\tilde{T}^{i} \tilde{h}^{i+\Delta \tilde{t}}}{\tilde{h}^{i}}+2 \tilde{T}^{i} \\
\tilde{C}_{j}^{i+\Delta \tilde{t}}=\frac{\tilde{D}_{j}^{i} D_{0} t_{0} \Delta \tilde{t}}{\left(\tilde{h}^{i} h_{0}\right)^{2}} \cdot \frac{\tilde{C}_{j+\Delta \tilde{z}}^{i}-2 \tilde{C}_{j}^{i}+\tilde{C}_{j-{ }^{\prime} \Delta \tilde{z}}^{i}}{\Delta \tilde{z}^{2}}+\tilde{C}_{j}^{i}
\end{gathered}
$$

The upper boundary is found using a ghost cell.

There is a trade-off between the accuracy and the stability of the numerical method. The dimensionless diffusion equation is the limiting term for the stability of this system. Thus, the stability criterion for the system can be expressed by the general stability criterion for FDM (Tannehill et al., 1997) on the diffusion equation as follows:

$$
\frac{\Delta \tilde{t}}{\Delta \tilde{z}^{2}} \leq \frac{1}{2 \max \left(\frac{\tilde{D} D_{0} t_{0}}{\left(\tilde{h} h_{0}\right)^{2}}\right)}
$$

Therefore, the selection of the resolution is done to get a stable and accurate enough solution.

\section{Results and Discussion}

The simulations for the composition of a $110 \mathrm{Wh}$ (around 30Ah), NMC811-G battery is carried out for selected process parameters. The film thickness, film temperature, solvent fraction, and the drying rate with time is plotted together in Figure 1. A hot air stream and a radiating heat source of the same temperature are used to test both the convective drying and infrared radiation drying. Only cathode drying with NMP as solvent is presented here for brevity, except otherwise stated.

As shown in Figure 1, The temperature of the film rapidly reaches the hot air temperature during infrared drying, and within 2 minutes for the convective drying, while the solvent evaporation is continued for about 90 minutes for both drying processes. The film thickness reached a constant value at the end of the drying period, where approximately $90 \%$ and $99 \%$ of the solvent is evaporated during convective and radiative drying, respectively. This is because the radiative drying gives a higher drying rate and shorter drying time than convective drying. However, it requires more energy for solvent removal $(Q)$ than the convective drying.

The general behavior of solvent removal and film thickness reduction as shown in Mesbah et al. (2014), can be seen here as well. Further, the film temperature transients and the drying rate are of a similar pattern to the model results given in Susarla et al. (2018). Moreover, Susarla et al. (2018) have used the capillary pressure together with the vapor pressure in the model, which gives high drying rates and low drying times. However, Ahmed et al. (2016) suggest that the cathode drying can be limited by the allowable NMP concentration in the dryer outlet air (1000-2000 ppm), which means that the cathode drying rate has an upper limit controlled by the dryer inlet air conditions and the initial film conditions. Further, Rollag et al. (2019) observed that increased electrode thickness and elevated drying temperatures cause crack formation in aqueous cathodes. These cracks were formed during the second stage of drying, which is driven by capillary forces. Therefore, improvements to the model to address these issues need to be thoroughly investigated in the future.

\subsection{Effect of Initial and Process Parameters}

The initial parameters such as the initial solvent mass fraction $C_{0}$ and the initial film thickness $h_{0}$ are defined by the type of battery that is being produced. However, the initial film temperature $T_{0}$, and the reference diffusion co- 

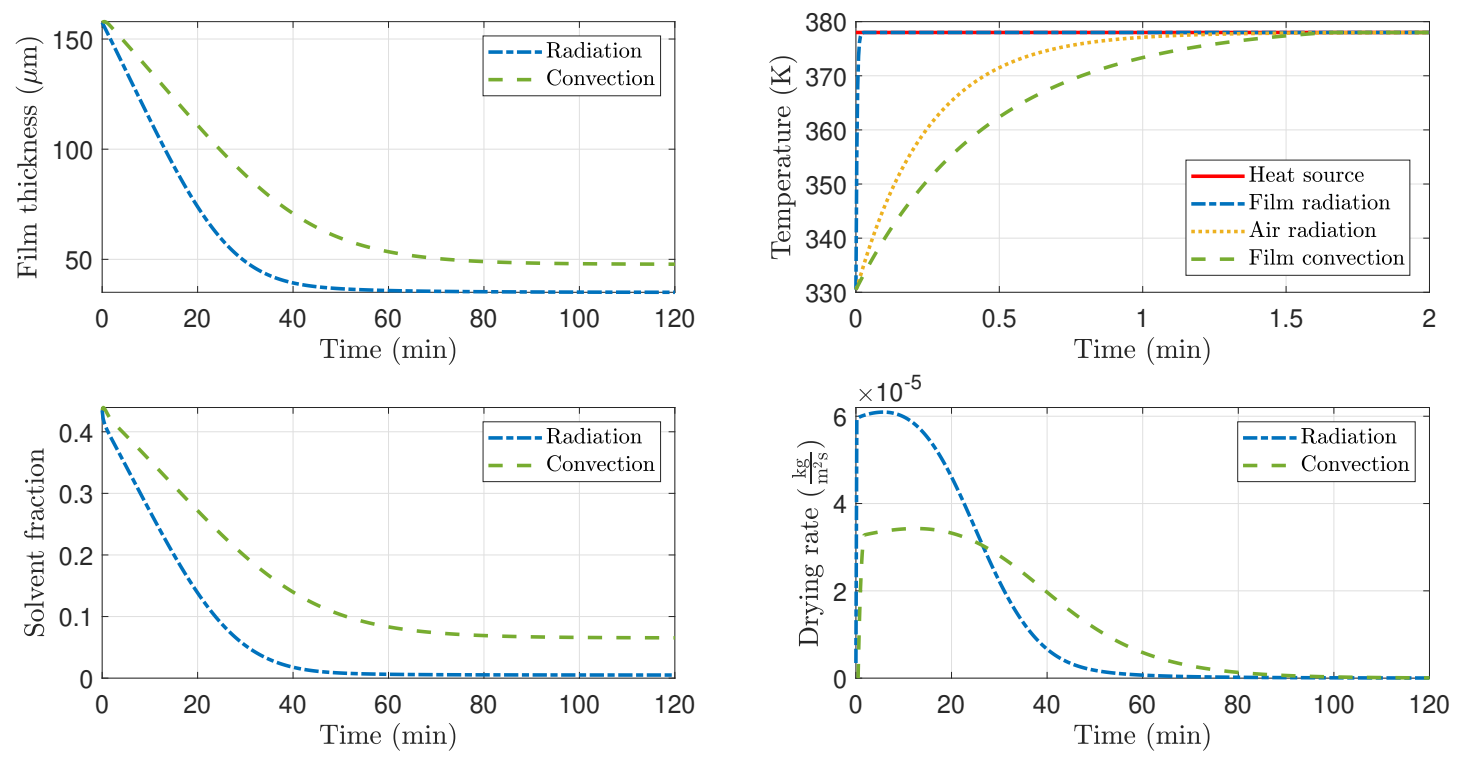

Figure 1. Film properties of the cathode during the convection and radiation drying processes.

efficient $D_{0}$ mainly depend on the indoor environmental conditions. The process parameters, which are the properties of the heat source (hot air or infrared heater) can be changed selectively. For convective drying, these parameters are the relative humidity $\varphi$, temperature $T_{\mathrm{a}}$, and the velocity $v$ of the hot air stream. For radiation, the temperature $T_{\mathrm{s}}$ and emissivity $\varepsilon$ of the heater are the parameters that can be changed.

To evaluate the effect of these parameters on the model outputs (convective drying model), a sensitivity analysis is conducted for all the parameters. The full range of input parameter values that is suitable for the selected battery type is considered. The changes in the model output values that happen due to the changes in these input parameter values are calculated, where only one parameter changes at a time. The sensitivities are determined as a percentage variation of the output for a percentage change of each initial or input parameter. Energy required for solvent evaporation ( $Q$ calculated by Equation 15) and the drying rate are selected as outputs. The spider plot for energy requirement is shown in Figure 2.

The most influential parameter on the energy consumption is the hot air temperature (red dash dotted line) as it rapidly changes the heat transfer. Having hotter air lowers the energy consumption. The energy requirement is highly sensitive to the initial thickness of the film upon entering the dryer (purple dash dotted line) along with the initial solvent concentration of the film (blue dash dotted line). The relative humidity (water) of the hot air is also affecting the heat transfer rate due to the direct correlation of humidity with the vapor pressure. Here, in this model, the relative humidity of hot air is considered to be less than 0.5 , since it is bounded by the maximum allowable limit of NMP in the outlet air of the dryer (Ahmed et al.,
2016). The other parameters have a low impact on energy consumption.

Further, the effect of the parameters on the rate of evaporation per area is calculated. All the parameters except the initial film temperature are plotted in Figure 3 since the initial film temperature has an insignificant impact on the model outputs. Change of the initial solvent concentration, the initial thickness, and the reference diffusion coefficient do not change the maximum drying rate that can be achieved. The initial solvent concentration also has little effect on the drying time. However, the initial thickness has a significant effect on the drying time, where thicker electrode coatings take a longer time to dry. Further, the lowest reference diffusion coefficient tends to have a considerably slower drying rate at the end of drying, which leads to longer drying time.

On the other hand, change of the hot air temperature, relative humidity, and air velocity change the maximum drying rate that can be achieved as well as the drying time. The direct effect of air temperature on the drying rate can

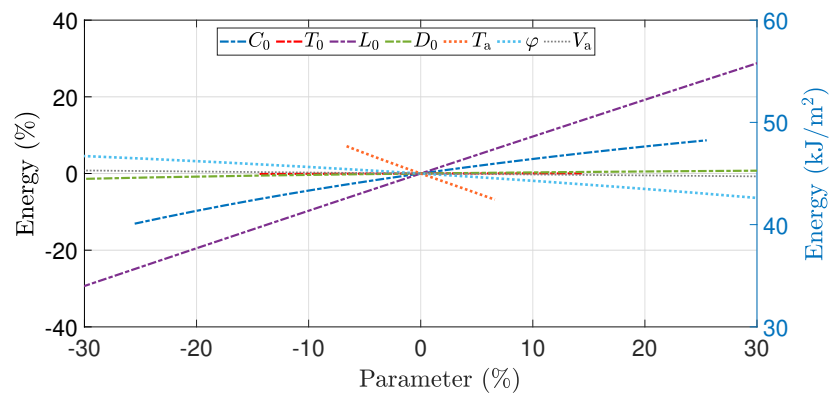

Figure 2. Effect of different initial and input parameters on the energy requirement. 
also be seen here. The higher the hot air temperature, the higher the heat transfer to the electrode, thus higher the drying rate and shorter the drying time. On the contrary, when the relative humidity is increased, the evaporation of solvent to the air reduces, hence the drying rate is decreased which results in longer drying time. Energy demand can be both reduced by increasing the humidity and increasing the hot air temperature. However, since fast drying is usually preferable in the industry, low humidity is needed. The increase of air velocity tends to increase the heat transfer similar to the behaviour of air temperature, thus increases the drying rate and reduces the drying time.

Since the air properties $\left(T_{\mathrm{a}}, \varphi, v\right)$ are controllable parameters, these can be used to obtain faster drying. However, these parameters would also directly contribute to the energy requirements. Therefore, optimum control of the air properties is needed for the drying process to be both efficient and less energy-intensive.

\subsection{Reduction of Energy Consumption}

Generally in convective drying, the use of hightemperature hot air would provide a high drying rate and low drying time, which would indicate low energy requirement for the solvent evaporation. However, a large amount of hot air is needed for this, and the heat requirement for producing this high-temperature hot air is significantly higher compared to the evaporation energy (Ahmed et al., 2016). Therefore, a multi-stage drying process is tested to utilize high-temperature hot air for a shorter time, such that the heating load would be reduced.

The film properties for a three-stage drying process where the hot air temperature is gradually increased in 2- steps are shown in Figure 4. The total drying time is significantly reduced compared to the previous results, and $99 \%$ of the solvent is removed. The energy required for the solvent evaporation is lower than the single-stage use of low-temperature hot air (see Figure 5). However, the evaporation energy for the 3-stage process is higher than for the single-stage use of high-temperature air.

The energy consumption for air heating is directly proportional to the inlet and outlet temperature difference of the air heater. Assuming a constant ambient air temperature and no re-circulation of hot air, this energy is calculated for different hot air temperatures. For single-stage drying with hot air temperatures of $353 \mathrm{~K}, 368 \mathrm{~K}$, and 388 $\mathrm{K}$, the energy needed for air heating is $392 \mathrm{MJ} / \mathrm{m}^{3}, 326$ $\mathrm{MJ} / \mathrm{m}^{3}$, and $276 \mathrm{MJ} / \mathrm{m}^{3}$, respectively. The 3-stage drying requires $198 \mathrm{MJ} / \mathrm{m}^{3}$, which is significantly less than the use of single-stage drying.

\subsection{Comparison with Reported Literature Values}

Considering the full range of all the parameters used in the single-stage drying, the distribution of energy requirement for convective air heating is calculated. Here, the drying of both the cathode and anode is added together. The results are compared with the reported values of energy for drying of industrial LIB plants of different capacities as shown in Figure 6.

The model values are closer to the values reported by Pettinger and Dong (2017) and Schünemann (2015), than the other values. However, the distribution of the model results has a high variation which resulted from the uncertainty in the used parameters. The range of the reported literature values is also high, due to various factors such
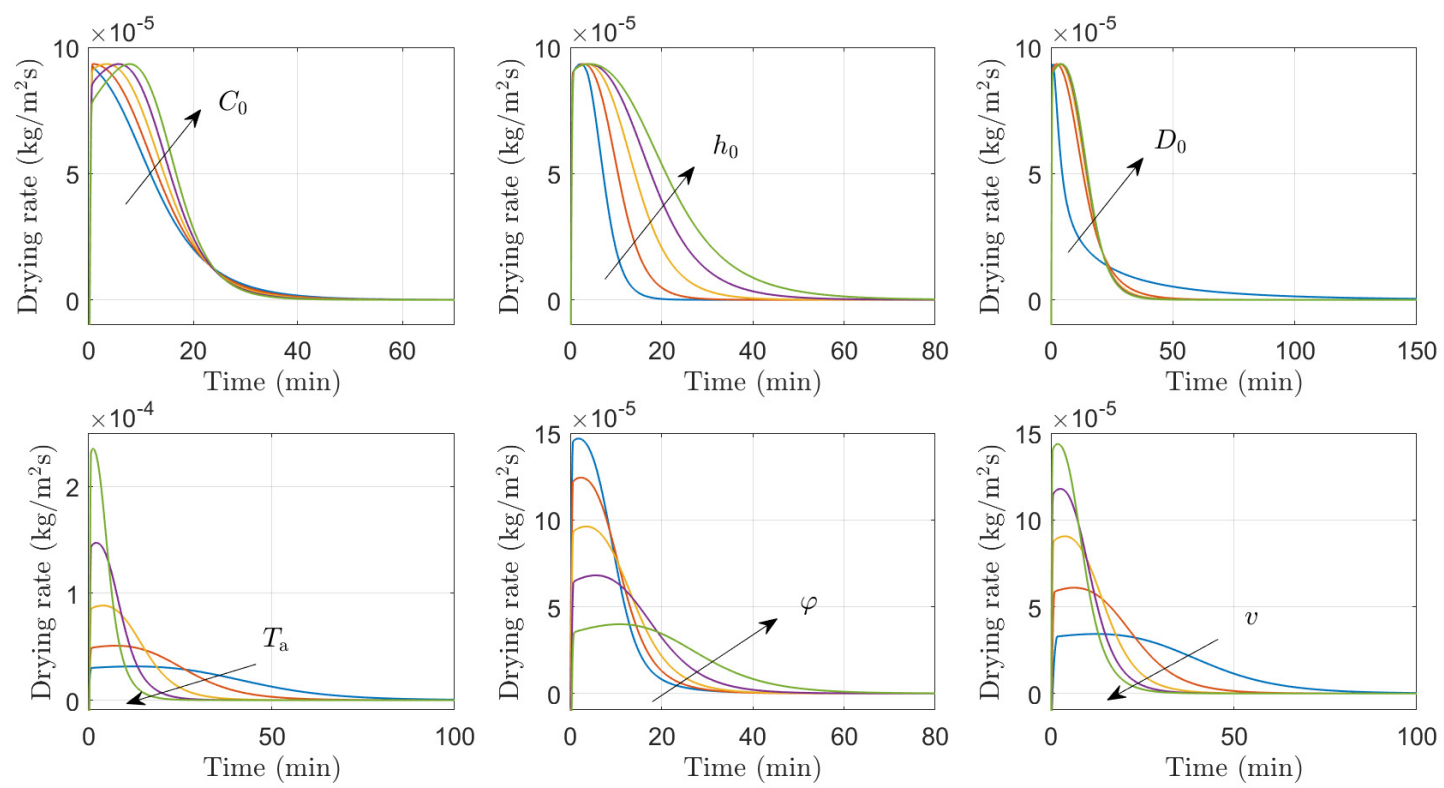

Figure 3. Effect of different initial and input parameters on the drying rate. $C_{0}$ : initial solvent mass fraction, $h_{0}$ : initial film thickness, $D_{0}$ : reference diffusion coefficient, $T_{\mathrm{a}}$ : hot air temperature, $\varphi$ : relative humidity of hot air, $v$ : velocity of hot air 

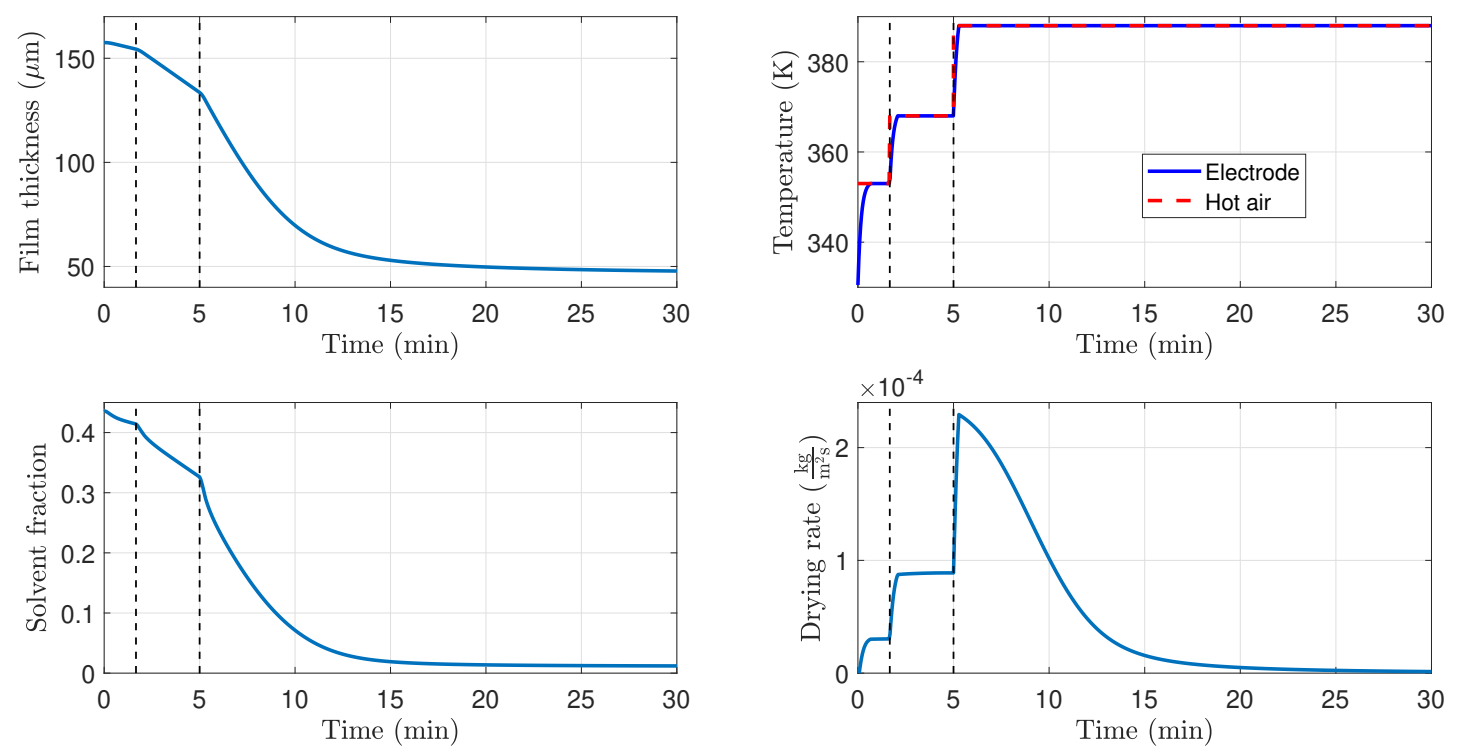

Figure 4. Film properties of cathode with the change of hot air temperature $T_{\mathrm{a}}$.

as the annual production capacity of the plant, different battery types, and various specific energies. Further, some of the reported values are for the combined process of both calendaring and drying, as well as 2 step drying processes where the remaining solvent is vacuum dried at a later stage after calendaring. The model results provide a benchmark, however, the model does not include energy for the NMP recovery unit. No details are given regarding the literature values, whether the energy values include a NMP recovery unit or not. Ahmed et al. (2016) shows in their model, that the energy loads for solvent recovery $(1969 \mathrm{~kW})$ are significant compared to the energy demand for air heating $(3752 \mathrm{~kW})$ and solvent evaporation (130 $\mathrm{kW}$ ).

Although the model needs improvements with the inclusion of porosity, and capillary-driven drying, the model has the capability of representing important electrode drying behaviours. Further, the model shows a good potential of being used in energy and process optimization, and process control design. Moreover, the model can be used to

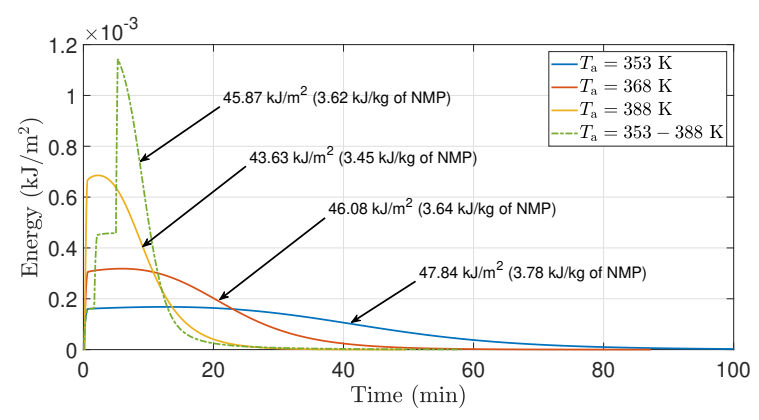

Figure 5. Energy required for the solvent evaporation at various hot air temperatures $T_{\mathrm{a}}$. The total energy (area of each curve) for each temperature is also stated. compare the energy demand or drying efficiency of different drying technologies, battery chemistries, and battery types. This would further provide the opportunity to modify the model and use it with life cycle assessment models to provide updated information on electrode drying. Thus, different process modifications can be tested and assessed by the energy requirement as well as the greenhouse gas emissions and cost. It would also contribute to benchmarking the energy demand for the LIB industry.

\section{Conclusions}

A simple, dynamic mathematical model for electrode drying is developed for the analysis of different drying technologies and energy requirement calculations. The input uncertainty through different parameters is decomposed to determine the most influential parameters on the model output. The heat source temperature, initial thickness, and solvent concentration of the electrode are the most sen-

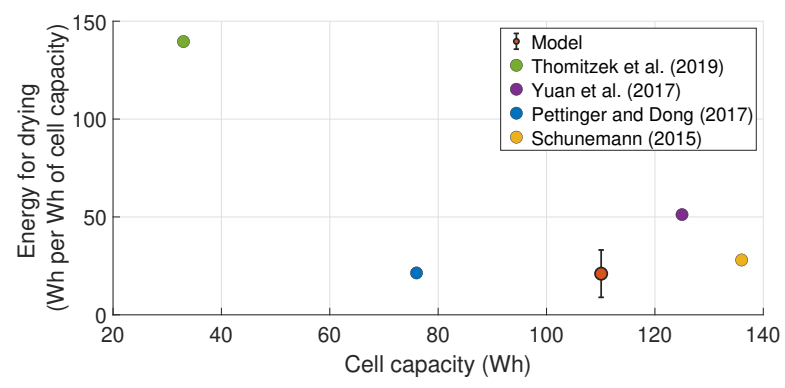

Figure 6. Comparison of energy consumption from drying model with reported studies of Schünemann (2015); Pettinger and Dong (2017); Yuan et al. (2017); Thomitzek et al. (2019). The model value is given with $\pm 2 \sigma$ variation. 
sitive parameters to both the drying rate and the energy consumption. Although the model is limited by the lack of porosity and capillary pressure, the results indicate the model sufficiently illustrates the general traits of an industrial electrode dryer. The results are compared with reported literature values.

Further, the use of multi-stage hot air distribution is shown to be less energy-intensive than the use of high temperature, single-stage hot air drying. The model shows a good potential of being used in energy optimization, and control design for drying processes. A further modified model can be used with life cycle assessment models. This could provide information on drying that changes with various drying technologies, energy recovery methods, and various LIB types in terms of energy requirement as well as the greenhouse gas emissions and cost.

\section{Acknowledgments}

The economic support from Freyr Battery AS and EIT Innoenergy SE (through project:02-2019-IP172-FREYR) is gratefully acknowledged.

\section{References}

Shabbir Ahmed, Paul A. Nelson, Kevin G. Gallagher, and Dennis W. Dees. Energy impact of cathode drying and solvent recovery during lithium-ion battery manufacturing. Journal of Power Sources, 322:169-178, 2016. doi:10.1016/j.jpowsour.2016.04.102.

Odne Stokke Burheim. Engineering Energy Storage. Academic press, 2017.

Qiang Dai, Jarod C. Kelly, Linda Gaines, and Michael Wang. Life cycle analysis of lithium-ion batteries for automotive applications. Batteries, 5(48), 2019. doi:10.3390/batteries5020048.

Linda Ager-Wick Ellingsen, Bhawna Singh, and Anders Hammer Strømman. The size and range effect: Life-cycle greenhouse gas emissions of electric vehicles. Environmental Research Letters, 11:1-9, 2016.

International Energy Agency. Global EV Outlook 2019. Technical report, International Energy Agency, 2019. URL www.iea.org/publications/reports/ globalevoutlook2019/.

Milad Asgarpour Khansary. Vapor pressure and flory-huggins interaction parameters in binary polymeric solutions. Korean Journal of Chemical Engineering, 33(4):1402-1407, 2016. doi:10.1007/s11814-015-0277-6.

Simon Davidsson Kurland. Energy use for GWh-scale lithiumion battery production. Environmental Research Communications, 2(1):012001, 2020. doi:10.1088/2515-7620/ab5e1e.

Arno Kwade, Wolfgang Haselrieder, Ruben Leithoff, Armin Modlinger, Franz Dietrich, and Klaus Droeder. Current status and challenges for automotive battery production technologies. Nature Energy, 3(4):290-300, 2018. doi:10.1038/s41560-018-0130-3.
Ali Mesbah, Ashlee N. Ford Versypt, Xiaoxiang Zhu, and Richard D. Braatz. Nonlinear model-based control of thinfilm drying for continuous pharmaceutical manufacturing. Industrial and Engineering Chemistry Research, 53(18):74477460, 2014. doi:10.1021/ie402837c.

Karl Heinz Pettinger and Winny Dong. When does the operation of a battery become environmentally positive? Journal of the Electrochemical Society, 164(1):A6274-A6277, 2017. doi:10.1149/2.0401701jes.

Kelsey Rollag, Daniel Juarez-Robles, Zhijia Du, David L. Wood, and Partha P. Mukherjee. Drying Temperature and Capillarity-Driven Crack Formation in Aqueous Processing of Li-Ion Battery Electrodes. ACS Applied Energy Materials, 2(6):4464-4476, 2019. doi:10.1021/acsaem.9b00704.

Jan-Hinnerk Schünemann. Modell zur Bewertung der Herstellkosten von Lithiumionenbatteriezellen. Sierke, 1st edition, 2015. ISBN 978-3-86844-704-0.

Naresh Susarla, Shabbir Ahmed, and Dennis W. Dees. Modeling and analysis of solvent removal during Li-ion battery electrode drying. Journal of Power Sources, 378(August 2017): 660-670, 2018. doi:10.1016/j.jpowsour.2018.01.007.

John C. Tannehill, Dale A. Anderson, and Richard H. Pletcher. Computational Fluid Mechanics and Heat Transfer. Taylor \& Francis, Washington, 2nd edition, 1997. ISBN 0070503281.

Matthias Thomitzek, Felipe Cerdas, Sebastian Thiede, and Christoph Herrmann. Cradle-to-gate analysis of the embodied energy in lithium ion batteries. In Procedia CIRP, volume 80, pages 304-309, 2019. doi:10.1016/j.procir.2019.01.099.

Chris Yuan, Yelin Deng, Tonghui Li, and Fan Yang. Manufacturing energy analysis of lithium ion battery pack for electric vehicles. CIRP Annals - Manufacturing Technology, 66(1): 53-56, 2017. doi:10.1016/j.cirp.2017.04.109. 